JOURNAL OF PHYSICAL CHEMISTRY C - NANOMATERIALS AND INTERFACES (ISSN: 1932-7447) 119(14): 7704-7710 (2015).

DOI: $10.1021 /$ acs.jpcc.5b01943 


\title{
DFT Study of Oxidation States on Pyrite Surface Sites
}

\author{
Tamás Rozgonyi ${ }^{1}$ and András Stirling ${ }^{2 *}$ \\ ${ }^{1}$ Institute of Materials and Environmental Chemistry, Research Centre for Natural Sciences \\ of the Hungarian Academy of Sciences, Budapest, Hungary and \\ ${ }^{2}$ Institute of Organic Chemistry, Research Centre for Natural Sciences \\ of the Hungarian Academy of Sciences, Budapest, Hungary
}

(Dated: March 17, 2015)

\begin{abstract}
Density functional calculations have been performed to study the interaction of oxygen with clean and defective pyrite (100) surfaces. Molecular adsorption states are predicted to undergo dissociation because atomic chemisorption is far more favorable thermodynamically for all surfaces. On the defect-free $\mathrm{FeS}_{2}$ surface molecular adsorption takes place on neighboring iron sites in a side-on fashion. S-adatoms react weakly with $\mathrm{O}_{2}$ whereas $\mathrm{S}$-vacancies bind very strongly molecular oxygen. Dissociative chemisorption on clean surface prefers the sulfur atoms to iron in sharp contrast to water adsorption. Reaction with atomic oxygen is thermodynamically preferred on the defect sites when they are available. Oxidation can fully eliminate the S-adatom sites by $\mathrm{SO}_{2}$ formation. The most favorable sites for oxidation are sulfur vacancies where $\mathrm{O}$ atoms can occupy the missing sulfur sites. The predicted oxidation states and their relative stabilities are in agreement with experiments and expected to assist the interpretation of XPS measurements.
\end{abstract}

\section{INTRODUCTION}

Oxidation of pyrite plays an essential role in various environmental and technological processes. ${ }^{1,2}$ Oxidation is more effective at the presence of water but employing dry $\mathrm{O}_{2}$ also yields oxidation products at high $\mathrm{O}_{2}$ dosages. ${ }^{2-7}$ Oxidation of pyrite present in minerals and coal during combustion processes in various industrial fields such as metallurgical processing, cement production or power production leads to harmful $\mathrm{SO}_{2}$ emission. ${ }^{8}$ A thorough understanding of the kinetics and themodynamics of pyrite oxidation is necessary to interpret and predict the decomposition and oxidation transformation of pyrite under different reaction conditions in industrial processes. ${ }^{8}$ Aqueous oxidation of pyrite leads to formation of large amount of protons giving rise to acidification of wastewater from mines. ${ }^{9}$ This is a serious problem and subsequent treatments require huge costs. ${ }^{10}$ Oxidation can also alter the surface properties of pyrite, in particular its wettability and hydrophilicity which can change the efficiency of flotation technologies for separating pyrite from other, more valuable minerals. ${ }^{2,11}$ Pyrite has also emerged as a promising material for photovoltaic applications. ${ }^{12}$ Facile oxidation is a problem also in this field because it can limit the potential scope of pyrite-based technologies.

A large number of studies have been carried out to investigate the surface properties of pyrite and in particular to identify and analyze various possible oxidation states. ${ }^{3-7,11,13-28}$ Atmospheric oxidation has been mostly studied by syn- chrotron and conventional XPS, $3,4,7,11,13-24$ but $\mathrm{IR},{ }^{6} \mathrm{UPS},{ }^{5,13} \mathrm{SEM}^{24}$ and $\mathrm{STM}^{5}$ have also been applied to identify surface oxidation products. Very few theoretical studies have been devoted to obtain atomic level understanding of the interaction of $\mathrm{O}_{2}$ with $\mathrm{FeS}_{2}$ surfaces. Hartree-Fock calculations have been used to identify oxidation products at iron sites of small $\mathrm{FeS}_{2}$ clusters representing different surface facets and sites. ${ }^{5}$ They predicted the dissociation of $\mathrm{O}_{2}$ is energetically more favorable then physisorption, in agreement with experiments despite the limitations of the selected method and models. Periodic calculations have been performed to elucidate the mechanism of the oxidation of the stoichiometric $\mathrm{FeS}_{2}$ surface in the presence of water. ${ }^{29}$ The results revealed the crucial role of the surface $\mathrm{O}^{2-}$ and $\mathrm{OH}^{-}$species in the oxidation cycles of the surface.

In this paper we compare the reactivity of clean and defective (100) $\mathrm{FeS}_{2}$ surface toward $\mathrm{O}_{2}$ and give a quantitative analysis of the various oxidation states formed by molecular and dissociative $\mathrm{O}_{2}$ chemisorption on the basis of periodic DFT calculations. Our aim is to provide atomistic details about the initial stages of the reaction of $\mathrm{FeS}_{2}$ with $\mathrm{O}_{2}$ without the presence of other potential reactant molecules. In particular we contrast the affinities of the surface iron and sulfur sites toward oxygen and compare the reactivities of the different sulfur sites. 


\section{MODELS AND METHODS}

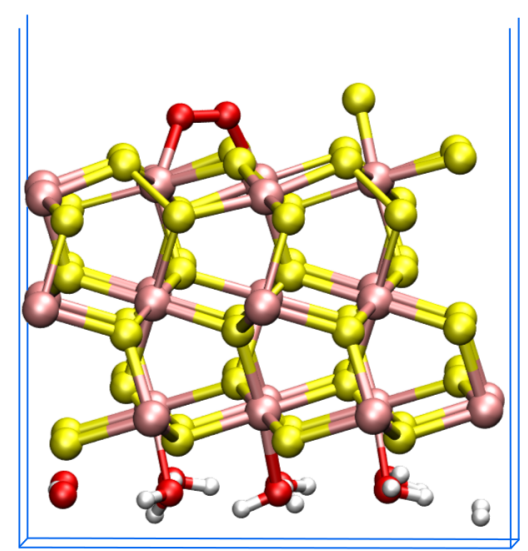

FIG. 1: Our simulation cell for a typical oxidation situation (molecular adsorption in the presence of a $\mathrm{S}$ adatom). Color coding: red - O, yellow - S, brown $\mathrm{Fe}$, white - H.

The calculations have been performed using the VASP code. $^{30}$ The electronic structure is described within the spin-polarized density functional framework employing the PW91 functional. ${ }^{31}$ A plane-wave basis set in conjunction with the PAW method ${ }^{32}$ has been used with a cutoff of $450 \mathrm{eV}$. The computed lattice constant of $\mathrm{FeS}_{2}$ is $5.415 \AA$ in nice agreement with experiment $(5.428 \AA) .{ }^{33}$ The calculated band gap is $0.80 \mathrm{eV}$ which is in good agreement with the experimental value of $0.95 \mathrm{eV} .^{12}$ For the adsorption states we have used a $2 \times 2$ orthorombic surface supercell with $a=b=10.830 \AA$ and $c=21.661 \AA$. The pyrite slab was 9 atomic layers thick and $\mathrm{a} \sim 10 \AA$ vacuum layer separates it from its periodic images in direction $c$. The bottom three layers have been kept fixed during the calculations. In order to prevent interactions between the two surfaces and to recover the bulk-like character of the lower layers the bottom iron atoms were terminated with water molecules. ${ }^{34}$ Without termination spin density can appear also at the bottom surface, but water termination can efficiently eliminate this artifact. The complete simulation model is depicted in Fig. 1. Dipole corrections ${ }^{35}$ accessible in VASP have been included for all systems. We have used a $3 \times$ $3 \times 1$ Monkhorst-Pack k-point set to sample the Brillouin zone which has been shown to be sufficient to obtain structural and energetic properties within the aspired accuracy $(0.001 \AA$ and 0.02 $\left.\mathrm{eV} / \AA^{2}\right) .^{36}$ Activation barriers have been calculated using the nudged elastic band method ${ }^{37,38}$ as implemented in the VASP code. Bader charges $^{39}$ have been calculated with the Bader Charge Analysis code developed by Henkelman et al. ${ }^{40}$

It is well-known that rupture of S-S bonds can occur during pyrite fracturing yielding surfaces with S-vacancy and S-adatom. In fact, experiments predict that $29 \%$ of the broken bonds are S$\mathrm{S}$ bonds. ${ }^{17}$ Therefore three different surfaces were considered. The stoichiometric (100) surface slab represents the perfect pyrite cleavage. For the defective (100) surfaces the slab models contain either a single S-vacancy or its fracture counterpart, a single S-adatom on an otherwise 5-coordinated surface iron within a unit cell.

\section{RESULTS}

\section{A. Molecular adsorption}

We have probed a large number of adsorption configurations on the three different surfaces. Both the molecular and dissociative states have been considered. First we discuss the molecular adsorption states of $\mathrm{O}_{2}$. The most stable configurations are depicted in Fig. 2.

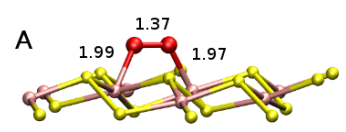

$-0.89$

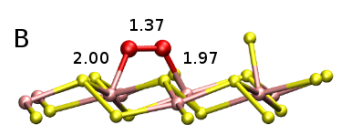

$-0.77$

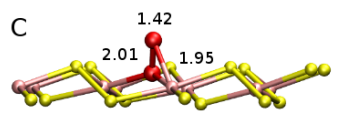

$-2.23$
FIG. 2: The most stable molecular adsorption configurations, $\mathrm{O}-\mathrm{O}$ and $\mathrm{Fe}-\mathrm{O}$ distances in $\AA$ and adsorption energies in $\mathrm{eV}$. $\mathrm{A}$ : on clean surface; $\mathrm{B}$ : on a surface with an S-adatom; C: on an S-vacancy. Only the top three $\mathrm{FeS}_{2}$ layers are shown for clarity. For color coding see Fig. 1.

On the clean surface the most stable molecular configuration for $\mathrm{O}_{2}$ is a side-on configuration, where two neighboring $\mathrm{Fe}$ atoms interact with the $\mathrm{O}_{2}$ molecule. The O-O bond is elongated with respect to the gas-phase $1.234 \AA$ to $1.374 \AA$ indicating peroxide formation which is further supported by a 0.3 e charge transfer to the $\mathrm{O}$ atoms from the pyrite slab as indicated by the Bader charges. Still, the ground state preserves the triplet spin state of the molecular oxygen. The adsorption energy is $-0.89 \mathrm{eV}$, slightly higher than that obtained in Ref. 29 (-0.72 eV) for the same adsorp- 
tion state. ${ }^{41}$ In contrast, Hartree-Fock (HF) calculations employing small clusters predicted $-0.4 \mathrm{eV}$ adsorption energy. ${ }^{5}$ Adsorption of $\mathrm{O}_{2}$ on a single surface iron in an end-on configuration is slightly less stable $(-0.72 \mathrm{eV}$, again slightly higher than that predicted in Ref. 29: $-0.61 \mathrm{eV}$ for this adsorption state). This form can be described as a superoxide state with a slightly elongated OO bond $(1.303 \AA)$. In this case an asymmetric charge distribution can be seen by inspecting the Bader charges: 0.3 e charge on the $\mathrm{O}$ atom binding to Fe whereas 0.2 e is localized on the farther $\mathrm{O}$ atom. Molecular adsorption on a sulfur site is not favorable. For this case we obtained a very small (-0.05 eV) adsorption energy which clearly indicates that molecular oxygen binds preferably to surface iron sites. When the surface features a defect (Fig. 2 B, C) the $\mathrm{O}_{2}$ can react with either the defect site or with a normal surface atom. In the case of an S-adatom, the most stable adsorption configuration is again the side-on configuration with $-0.77 \mathrm{eV}$ adsorption energy. This state is quite similar to that observed for the clean surface, although with a slightly smaller adsorption energy. This $-0.12 \mathrm{eV}$ discrepancy may be due to a possible interaction with the S-adatom, but may also be ascribed to the typical technical limitations expected in the calculations. End-on adsorption on a single surface Fe is less favorable by $0.1 \mathrm{eV}(-0.64 \mathrm{eV})$. This difference is very similar to what we found for the clean surface and points to a limited effect of the S-adatom on the $\mathrm{O}_{2}$ affinity of the neighboring irons. Even less favorable is the molecular adsorption on an S-adatom yielding only $-0.4 \mathrm{eV}$ reaction energy. In contrast, when an $\mathrm{S}$-vacancy site is available, the $\mathrm{O}_{2}$ molecule forms a very stable peroxo-complex with it releasing $2.23 \mathrm{eV}$ reaction energy. Its bond length is increased to $1.416 \AA$, only slightly shorter than a typical peroxo bond (1.48 $\AA$ ). The large stability of this state is due to the three simultaneous Fe$\mathrm{O}$ bonds formed between the $\mathrm{O}_{2}$ and the vacancy (Fig. 2C). Indeed, one of the oxygen atoms occupies the position of the S-vacancy forming bonds with the two neighboring 4-coordinated iron ions, whereas the other oxygen atom forms bond with the closer iron site. This adsorption state is spinunpolarized, in accordance with the large number of oxygen bonds.

\section{B. Dissociative adsorption on clean surface}

Before discussing the dissociative forms let us compare the reactivity of the sulfur and iron sites of the clean surface. To this end we considered the
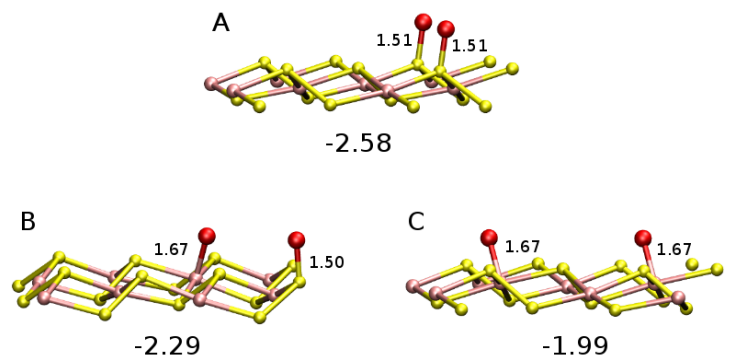

FIG. 3: The most stable dissociative configurations on clean $\mathrm{FeS}_{2}$ surface with adsorption energies in $\mathrm{eV}$ and with the relevant $\mathrm{S}-\mathrm{O}$ and $\mathrm{Fe}-\mathrm{O}$ bond lenghts in $\AA$. A: on S sites; B: on $\mathrm{S}$ and Fe sites; C: on iron sites. Only the top three $\mathrm{FeS}_{2}$ layers are shown for clarity. For color coding see Fig. 1.

adsorption of a single oxygen atom on both sites and compare the reaction energies of the hypothetical processes: $\mathrm{X}_{\text {surf }}+1 / 2 \mathrm{O}_{2} \longrightarrow \mathrm{X}_{\text {surf }}-\mathrm{O}$. The reaction energies are $-1.07 \mathrm{eV}$ and $-1.37 \mathrm{eV}$ with a surface iron and sulfur atom, respectively. (The reaction energies implicitly include the dissociation of the $\mathrm{O}_{2}$ molecule, ie. as indicated by the half mole of $\mathrm{O}_{2}$ in the above equation. Interaction with a single $\mathrm{O}$ atom would yield $\mathrm{a} \sim-2.6$ $\mathrm{eV}$ higher energy release. ${ }^{43}$ ) This $0.3 \mathrm{eV}$ difference indicates that oxidation of the exposed sulfur atoms is preferred. The oxidation of the iron site results in the formation of a Fe-O double bond and triplet state implying a bonding mechanism which has been found earlier for the S-adatom ${ }^{34,42}$. This is not surprising as both $\mathrm{S}$ and $\mathrm{O}$ are chalcogens and similar bonding situations were already seen in analogous chemical environment. ${ }^{44}$ In fact double bonds are predicted for these situations ${ }^{29,34}$ and accordingly the calculated Fe-O bond length is $1.671 \AA$. This value is much smaller than a typical Fe-O single bond $(\sim 2.1 \AA) \cdot{ }^{29,46-48}$ and falls in the range of the double $\mathrm{Fe}-\mathrm{O}$ bonds observed in ferryl compounds. ${ }^{49}$ The S-O bond is spin-free and has a $1.503 \AA$ length indicating double bond also for this case.

Further insights can be obtained from the density of states (DOS-s) of the relevant atoms. Fig. 4 displays the DOS-s of the oxidized sites. For comparison we also show the DOS-s of the normal surface Fe and S atoms (Fig. $4 \mathrm{~A}$ ). The DOS-s from the surface atoms have been discussed several times earlier. ${ }^{34,42}$. It has been shown that in the binding energy region of $-16--11 \mathrm{eV}$ the sulphur s contributions can be found. Between -7.5 and $-2 \mathrm{eV}$ the contributions for the Fe-S $\sigma$ bonds can be localized, followed by the iron $3 d$ states till the edge of the valence band. Oxidation of a sur- 


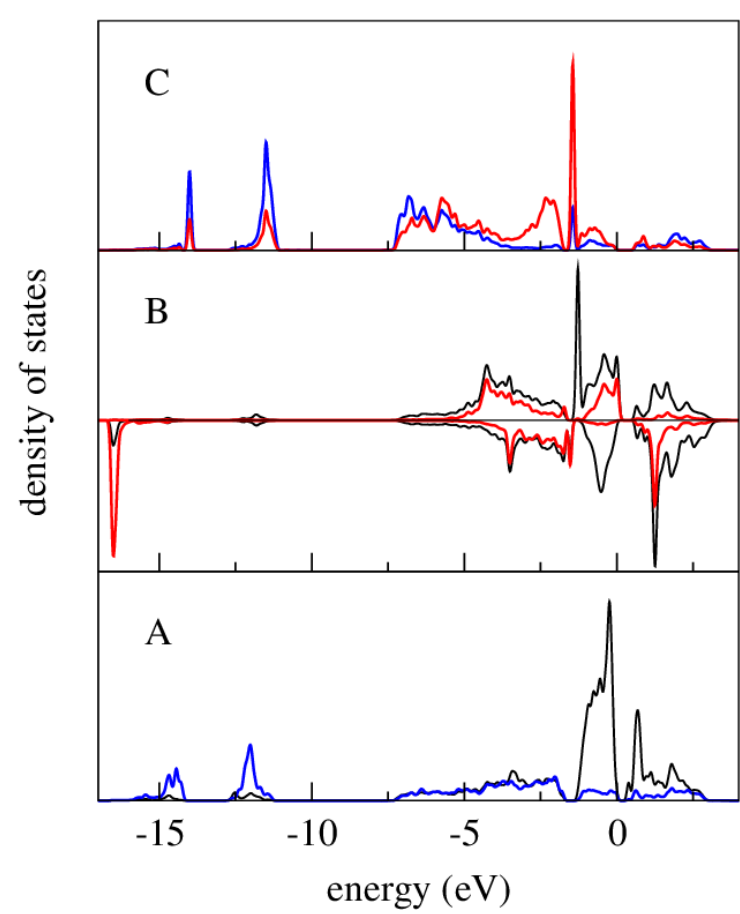

FIG. 4: Atomic DOS curves. A: normal surface Fe and $\mathrm{S}$ atoms. $\mathrm{B}$ : $\mathrm{Fe}$ and $\mathrm{O}$ atoms of the Fe-O monoxide unit. This form is spin-polarized, so both spin states are plotted with opposite signs. C: S and O atoms of the S-O monoxide unit. The Fermi levels are set to zero in all cases. Color coding: black: Fe; blue: S; red: $\mathrm{O}$.

face iron results in new bands at the $3 d$ region: the partially occupied antibonding $\pi^{*}$ orbitals can be identified close to the gap whereas the two doubly occupied pi orbitals are found $c a .3 \mathrm{eV}$ lower (Fig. $4 \mathrm{~B})$. It is also seen that a minority spin $3 \mathrm{~d}$ orbital becomes empty due to the oxidation state change of iron. Additional changes at the lower energy $\mathrm{Fe}-\mathrm{S} \sigma$ region are due to the mixing of the Fe-O and Fe-S $\sigma$ orbitals. As Fig. $4 \mathrm{C}$ shows, the oxidation of a surface $\mathrm{S}$ is accompanied by the mixing of the S-O $\sigma$ orbitals with the original Fe-S and $\mathrm{S}-\mathrm{S}$ bonds. The sharp new band around $-1.4 \mathrm{eV}$ indicates the formation of a S-O $\pi$ bond.

Why does oxygen atom have higher affinity toward surface sulfur than iron? To explain the origin of this difference we note that surface sulfur atoms have doubly occupied dangling bonds (lone pairs) whereas surface iron atoms hosts empty dangling orbitals. ${ }^{2,46,50}$ Binding of oxygen atom on the surface is a redox process and as such it is always accompanied with the reduction of the oxygen and oxidation of its bonded counterpart (electron transfer to the $\mathrm{O}$ atom). A surface sulfur atom can easily form bond with the oxygen atom via its lone pair. In contrast, bond formation with a surface iron requires that the $\mathrm{Fe}$ ion form an
$\mathrm{Fe}(\mathrm{IV})$ ion by donating electrons via its originally empty dangling orbital to the $\mathrm{O}$ atom (see also Fig. 4 B). A different mechanism occurs when water (or $\mathrm{H}_{2} \mathrm{~S}$ ) is adsorbed on the surface (Lewis acid-base reaction): the empty dangling orbitals on iron can easily bind the water molecules by overlapping with one of the lone pairs of the water oxygens, whereas sulfur atoms repel water because of the repulsive interaction between the fully occupied lone pairs. ${ }^{46,47}$

Before discussing the various dissociative scenarios we note that the calculated barrier of the migration of an $\mathrm{O}$ atom from a normal iron to a sulfur site is $0.58 \mathrm{eV}$. Using the simple Arrhenius approximation for the rate: $k=$ $\nu \exp \left(-E_{a c t} / R T\right)$ where $\nu$ is a typical $\mathrm{Fe}=\mathrm{O}$ frequency ${ }^{45}$ of $800 \mathrm{~cm}^{-1}$ we obtain at room temperature $\sim 0.3 \mathrm{~ms}$ for the time-scale of this migration $\left(k \cong 3800 s^{-1}\right)$ which is faster than a typical experimental time-scale. As the sulfur oxidation is more favorable by $-0.3 \mathrm{eV}$, the reverse barrier is larger $(0.88 \mathrm{eV})$ and implies a much less likely process.

Turning to the dissociation of $\mathrm{O}_{2}$ on $\mathrm{FeS}_{2}$ surfaces we considered first the clean, defect-free surface. Three different situations have been probed: oxidation of $\mathrm{Fe}$ atoms, oxidation of $\mathrm{S}$ atoms and mixed (Fe and S) oxidation. The most stable dissociative configurations obtained for these cases are shown in Fig. 3. We note that various other configurations were also calculated but turned to be less favorable. The calculated energies clearly show that dissociative chemisorption is thermodynamically far more favorable then molecular adsorption. This implies that molecular $\mathrm{O}_{2}$ adsorbed on the surface is expected to undergo dissociation. As it can be anticipated on the basis of the individual reaction energies obtained for a single oxygen atom, the simultaneous oxidation of two sulfur atoms is the most favorable process $(-2.58 \mathrm{eV})$, whereas the oxidation of iron sites yields the smallest reaction energy $(-1.99 \mathrm{eV})$. In all cases the reaction heats are very close to the sum of the oxidation energies of the individual sites indicating small interaction between the adsorbates. This implies that the so-called proximity effect predicted and observed in simultaneous $\mathrm{O}_{2}$ and water adsorption cannot be applied in the present $_{\text {cases. }}{ }^{4,5,51}$ In line with this finding the $\mathrm{Fe}-\mathrm{O}$ and $\mathrm{S}-\mathrm{O}$ bond lengths are the same as those found for the isolated oxides (within a few thousandths of an $\AA$ ) in all cases. Note that HF calculations using small (27-atom) cluster resulted in a much larger adsorption energy $(-18.6 \mathrm{eV})$ on iron sites. ${ }^{5}$ This huge discrepancy can be attributed to the limitations of the HF method and the cluster approximation. 
Regarding the kinetics of the formation of the various stable states on the clean surface we found that the dissociation of the $\mathrm{O}_{2}$ molecule in its stable side-on configuration (Fig. $2 \mathrm{~A}$ ) requires $0.3 \mathrm{eV}$ activation energy and yields the oxidation of two neighboring Fe sites. The stable states shown in Fig. 3 are formed by subsequent $\mathrm{O}$ migrations, which require $0.58 \mathrm{eV}$ activation energy as discussed above. This implies that the rate determining step leading to these stable oxidation states is the migration, not the dissociation of the adsorbed $\mathrm{O}_{2}$ molecule.

\section{Dissociative adsorption on defective surfaces}

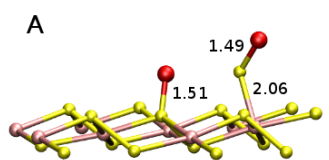

$-3.17$

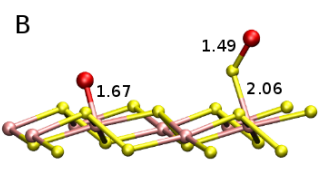

$-2.88$

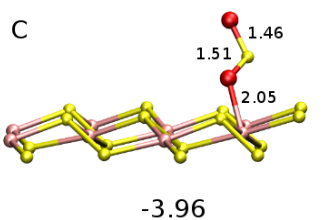

FIG. 5: The most stable dissociative configurations, the relevant Fe-S, Fe-O and $\mathrm{S}-\mathrm{O}$ distances in $\AA$ and adsorption energies in $\mathrm{eV}$ on defective $\mathrm{FeS}_{2}$ surface with a sulfur adatom. A: on a sulfur site and on the sulfur adatom; B: on an iron site and on the sulfur adatom; $\mathrm{C}$ : adsorbed $\mathrm{SO}_{2}$ on an iron site. Only the top three $\mathrm{FeS}_{2}$ layers are shown for clarity. For color coding see Fig. 2.

When a sulfur adatom is present, the surface becomes significantly more reactive. This is partly due to the presence of unpaired spin dominantly localized on the adatom site ${ }^{34,42}$ but also to the more reduced, -2 oxidation state of the exposed sulfur atom as compared to the -1 state of the normal surface and bulk sulfur atoms. ${ }^{34}$. Indeed measurements have convincingly indicated this undercoordinated $\mathrm{S}$ site to be the most sensitive to oxidation. ${ }^{7,19-23,25,52}$ The most favorable process in the presence of the S-adatom is the formation of an $\mathrm{SO}_{2}$ molecule with the defect sulfur releasing substantial reaction heat $(-3.96 \mathrm{eV})$. The product $\mathrm{SO}_{2}$ of this process is adsorbed on the iron site and its desorption would require $0.72 \mathrm{eV}$ energy investment which is available from the preceding oxidation. This desorption can be irreversible in prac- tice consuming all the S-adatom sites from the surface. Simultaneous oxidation of the S-adatom and either a sulfur or an iron site is less favorable. The energy difference between these two possible products are in agreement with that obtained for the monooxide states $(\sim 0.3 \mathrm{eV})$. The higher reactivity of the S-adatom is also reflected by its smaller bond length formed with a single oxygen $(1.494 \AA$ ). In contrast, the other O atom forms the typical oxide bonds with the normal surface atoms as seen before. In case of $\mathrm{SO}_{2}$ formation the adsorption slightly lengthens the S-O bond interacting with the surface iron (1.514 $\AA$ ), whereas the other becomes even stronger (1.464 $\AA)$. The $\mathrm{SO}_{2}$ adsorption takes place via a single Fe-O bond $(2.051 \AA)$. The calculated activation energies again show that the migration of $\mathrm{O}$ atoms on the surface are the rate determining steps leading to the formation of these states from the molecular adsorption states. Indeed dissociation of an $\mathrm{O}_{2}$ molecule in its preferred configuration in the presence of an $\mathrm{S}$ adatom (Fig. $2 \mathrm{~B}$ ) requires only $0.18 \mathrm{eV}$ activation energy and leads to the immediate oxidation of the $\mathrm{S}$ adatom, whereas the other $\mathrm{O}$ atom rests on the iron sites (cf. Fig. $5 \mathrm{~B}$ ). We have also calculated the activation energy necessary to the direct oxidation of the S-adatom to $\mathrm{SO}_{2}$ when the $\mathrm{O}_{2}$ molecule is adsorbed on it. This barrier is $0.39 \mathrm{eV}$ which is again reasonably small and supports the high reactivity of these sites.

From the various oxidation forms occurring on this surface we have selected the mono-oxidized S-adatom for DOS calculation because the electronic structure of this configuration has some peculiarities Fig. 6 B shows the atomic DOS-s for the relevant Fe-S-O unit. We can see that the original Fe-S $\pi$ and $\pi^{*}$ orbitals $^{34}$ overlap with the new S-O $\pi$ orbitals (around $-5.5 \mathrm{eV}$ and $-1.1 \mathrm{eV}$ ). Similarly we can notice the rehybridization of the Fe-S and S-O $\sigma$ orbitals (around $-11 \mathrm{eV}$ ). In other words the DOS-s indicate several 3-center bonds for this Fe-S-O unit.

A sulfur vacancy on the (100) $\mathrm{FeS}_{2}$ surface has also been measured to be quite reactive. ${ }^{7,14,15,18-23,25,50}$ Although the vacancy is shown to be spin-unpolarized, ${ }^{34,48}$ the presence of two 4-coordinated iron ions significantly increases the reactivity of the site. ${ }^{48}$ In addition, the undercoordinated $\mathrm{S}$ atom remaining in the vacancy due to the rupture of a S-S bond is also shown to be in its possible most reduced, -2 oxidation state $^{34,50,52,53}$ implicating also higher reactivity. The possible structural variants of the dissociative adsorption of $\mathrm{O}_{2}$ involving the vacancy are shown in Fig. 7. One of the oxygens occupies 


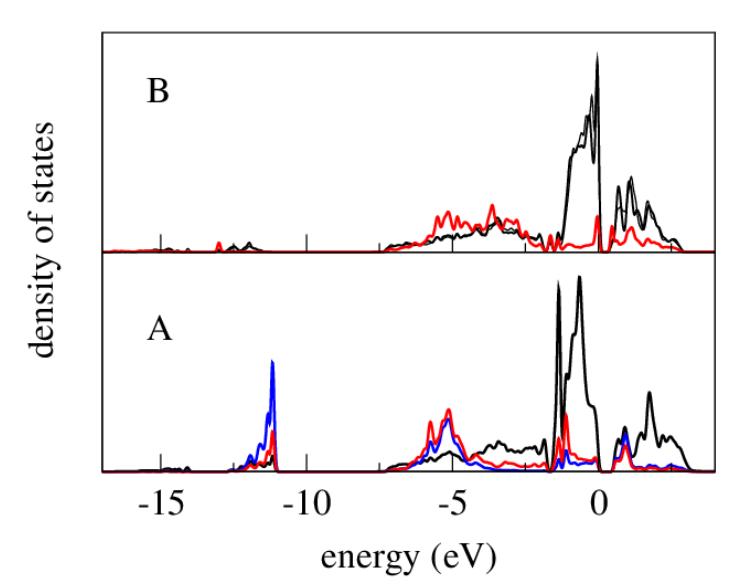

FIG. 6: Atomic DOS curves. A: Contribution of Fe, S and $\mathrm{O}$ atoms in case of a sulfur adatom; B: Contribution from the neighbor irons and the $\mathrm{O}$ atom in case of a sulfur vacancy. The Fermi levels are set to zero in all cases. Color coding: see Fig. 4.

the vacancy site saturating partially the coordination spheres of the vacancy irons. Comparing the two situations when the other $\mathrm{O}$ atom binds to an iron we find that the vacancy iron has very similar affinity toward oxidation to a regular surface iron site ( $c f$. Figs $7 \mathrm{~A}$ and $\mathrm{C}$ ). The calculated energy difference $(\sim-0.1 \mathrm{eV})$ may be attributed to DFT errors. Clearly more favorable is the situation when a surface sulfur atom is oxidized. This yields an additional $0.3 \mathrm{eV}$ energy release in accordance with the higher reactivity of the surface sulfur shown earlier. While the oxidation of the normal surface atoms shows the same bonding pattern as seen earlier, the oxygen atom in the vacancy forms quite stable bonds with the three neighboring atoms. In fact it becomes threecoordinated by covalent bonds with the two iron and the sulfur atoms. The distribution of the possible Fe-O and S-O bond lengths (1.85-2.21 $\AA$ and 1.71-2.4 $\AA$, respectively, obtained for the various possible configurations) indicates a certain degree of flexibility for the 3-coordinated $\mathrm{O}$ atom within the vacancy. It is interesting to see from Fig. 6 $\mathrm{B}$ that the $\mathrm{O}$ atom in the vacancy shows quite similar electronic behavior to the original $\mathrm{S}$ atom: the main contributions are in the original $\mathrm{Fe}-\mathrm{S} \sigma$ region with only a very small p-d overlap near to the gap ( $c f$. Fig $4 \mathrm{~A}$ ).

The calculated activation energy of the dissociation of an $\mathrm{O}_{2}$ molecule in a S-vacancy (see Fig. $2 \mathrm{C}$ ) is $0.16 \mathrm{eV}$. This process results in the oxidized state depicted in Fig. 7 A. Subsequent O migrations will lead to the oxidation of a surface $\mathrm{S}$ which are in fact the rate determining steps with $0.58 \mathrm{eV}$ activation barriers as pointed out in the earlier cases.
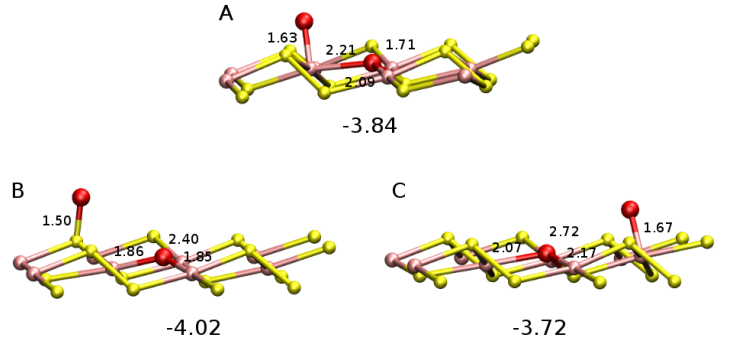

FIG. 7: The most stable dissociative configurations, relevant $\mathrm{Fe}-\mathrm{O}$ and $\mathrm{S}-\mathrm{O}$ distances in $\AA$ and adsorption energies in $\mathrm{eV}$ on defective $\mathrm{FeS}_{2}$ surface with a sulfur vacancy. A: occupying the vacancy site and an empty site on a neighboring iron; B: oxygen atoms coordinating to the vacancy and to a normal sulfur; C: oxygen atoms bonding to the vacancy and a normal surface iron. Only the top three $\mathrm{FeS}_{2}$ layers are shown for clarity. For color coding see Fig. 2.

\section{DISCUSSION}

A couple of general conclusions can be drawn from the calculations. First, however, we note that the present calculations intend to look into the very first step of dry oxidation of pyrite surfaces: it represents the exposure of the (100) surface to an $\mathrm{O}_{2}$ dosage of 0.3 langmuir. ${ }^{54}$ Therefore, comparison of the present theoretical results with the experimental spectra obtained for larger doses must be done with care. Both molecular and dissociative $\mathrm{O}_{2}$ adsorption prefers the $\mathrm{S}$-vacancy sites most. However, when these sites are not available, the situation is different. Oxygen binds molecularly to iron sites, but after dissociation it clearly prefers the sulfur sites on the surface. Experiments have long indicated that the (100) pyrite surface is very stable and first the most reduced sulfur components are oxidized in the presence of $\mathrm{O}_{2} \cdot{ }^{6,17,19-23}$. Calculations have already shown that both sulfur defect sites have a nominal oxidation number of -2 , whereas the other $\mathrm{S}$ atoms are in their -1 oxidation state. ${ }^{34,42}$ Therefore the calculated high reactivity of these sites is in perfect agreement with experiment. However, there is a disagreement in the literature regarding the reactivity differences of the various sulfur and iron sites. Several studies suggest that oxidation of surface sulfur sites precedes the oxidation of iron site. ${ }^{17,19,22}$ Others propose that iron sites react faster than sulfur sites. ${ }^{2,5,6,25}$ At the origin of this controversy can be the sensitivities of the XPS analysis $^{25}$ and other factors (e.g the time resolution of the measurements to capture fast reaction steps). We can reconcile theory and experiment if 
we recall that in all surfaces considered here the molecular adsorptions resulted in Fe-O bond formations. Only in the case of S-vacancy we could obtain S-O bond as well. In contrast the dissociative adsorptions yielded both sulfur and iron oxidations in the presence of surface defects. The calculated activation energies showed that these processes are feasible even at room temperature. Therefore the proposed sequence of molecular adsorption on surface iron sites and S-vacancy sites followed by dissociative adsorption producing oxidized iron and sulfur states brings theory and experiment into agreement.

\section{CONCLUSIONS}

In summary our periodic DFT calculations revealed the following plausible sequence of reaction steps for the incipient oxidation of pyrite surface: $\mathrm{O}_{2}$ in molecular form adsorbs on iron sites in a side-on configuration before finding the most favorable S-vacancy sites. After dissociation oxidation of sulfur sites are preferred although oxidation of iron sites are also favored thermodinamically. In particular the oxidation of S-adatom and saturation of S-vacancies are highly favorable at the initial stage of oxidation. This sequence is in agreement with experimental observations and provides important insight into the incipient oxidation of pyrite.

\section{ACKNOWLEDGMENT}

Support of OTKA Grant K101115 and computational resources provided by NIIF Supercomputer Center are acknowledged. 


\section{REFERENCES}

${ }^{1}$ Murphy, R.; Strongin, D. R. Surface Reactivity of Pyrite and Related Sulfides. Surf. Sci. Rep 2009, 64, 1-45.

${ }^{2}$ Chandra, A. P.; Gerson, A. R. The Mechanisms of Pyrite Oxidation and Leaching: A Fundamental Perspective. Surf. Sci. Rep 2010, 65, 293-315.

${ }^{3}$ Guevremont, J. M.; Bebie, J.; Elsetinow, A. R.; Strongin, D. R.; Schoonen, M. A. A. Reactivity of the (100) Plane of Pyrite in Oxidizing Gaseous and Aqueous Environments: Effects of Surface Imperfections. Environ. Sci. Technol. 1998, 32, 37433748.

${ }^{4}$ Guevremont, J. M.; Elsetinow, A. R.; Strongin, D. R.; Bebie, J.; Schoonen, M. A. A. Structure Sensitivity of Pyrite Oxidation: Comparison of the (100) and (111) Planes. Am. Mineral. 1998, 83, $1353-1356$

${ }^{5}$ Rosso, K. M.; Becker, U.; Hochella Jr., M.F. The Interaction of Pyrite 100 Surfaces with $\mathrm{O}_{2}$ and $\mathrm{H}_{2} \mathrm{O}$ : Fundamental Oxidation Mechanisms. Am. Mineral. 1999, 84, 1549-1561.

${ }^{6}$ Usher, C. R.; Paul, K. W.; Narayansamy, J.; Kubicki, J. D.; Sparks, D. L.; Schoonen, M. A. A.; Strongin, D. R. Mechanistic Aspects of Pyrite Oxidation in an Oxidizing Gaseous Environment: An in Situ HATR-IR Isotope Study. Environ. Sci. Technol. 2005, 39, 7576-7584.

7 Chandra, A. P.; Gerson, A. R. Pyrite $\left(\mathrm{FeS}_{2}\right)$ Oxidation: A Sub-Micron Synchrotron Investigation of the Initial Steps. Geochim. Cosmochim. Acta 2011, 75, 6239-6254.

${ }^{8} \mathrm{Hu}$, G.; Dam-Johansen, K.; Wedel, S.; Hansen, J. P. Decomposition and Oxidation of Pyrite. Prog. Energy Combust. Sci. 2006, 32, 295-314.

9 Lowson, R. T. Aqueous Oxidation of Pyrite by Molecular Oxygen. Chem. Rev. 1982, 82, 461-497.

10 Evangelou, V. P.; Zhang, Y. L. A Review: Pyrite Oxidation Mechanisms and Acid Mine Drainage Prevention. Crit. Rev. Environ. Sci. Technol. 1995, 25, 141-199.

11 Buckley, A. N.; Woods, R. The Surface Oxidation of Pyrite. App. Surf. Sci. 1987, 27, 437-452.

12 Ennaoui, A.; Fiechter, S.; Pettenkofer, C.; AlonsoVante, N.; Büker, K.; Bronold, M.; Höpfner, C.; Tributsch, H. Iron Disulfide for Solar Energy Conversion. Sol. Energy Mater. Sol. Cells 1993, 29, 289-370.

13 Eggleston, C. M.; Ehrhardt J.--J.;Stumm, W. Surface Structure Controls on Pyrite Oxidation Kinetics: An XPS-UPS, STM and modeling study. Amer. Miner. 1996, 81, 1036-1056.

${ }^{14}$ Karthe, S.; Szargan, R.; Suoninen, E. XPS Study of the Sulphur 2p Spectra of Pyrite. App. Surf. Sci. 1993, 72, 157-170.

15 Nesbitt, H. W.; Muir, I. J. X-ray Photoelectron Spectroscopic Study of a Pristine Pyrite Surface Reacted with Water Vapour and Air. Geochim.
Cosmochim. Acta 1994, 58, 4667 (1994).

16 Schaufu $\beta$, A. G.; Nesbitt, H. W.; Kartio, I. ; Laajalehto, K.; Bancroft, G. M.; Szargan, R. Reactivity of Surface Chemical States on Fractured Pyrite. Surf. Sci. 1998, 411, 321-328.

17 Schaufu $\beta$, A. G. ; Nesbitt, H. W.; Kartio, I.; Laajalehto, K.; Bancroft, G. M.; Szargan, R. Incipient Oxidation of Fractured Pyrite Surfaces in Air. $J$. Electron Spec. Rel. Phen. 1998, 96, 69-82.

18 Nesbitt, H. W.; Scaini, M. J.; Höchst, H.; Bancroft, G. M.; Schaufuss, A. G.; Szargan, R. Synchrotron XPS Evidence for $\mathrm{Fe}^{2+}-\mathrm{S}$ and $\mathrm{Fe}^{3+}{ }_{-} \mathrm{S}$ Surface Species on Pyrite Fracture-Surfaces, and Their 3D Electronic States. Am. Mineral. 2000, 85, 850857.

19 Uhlig, I.; Szargan, R.; Nesbitt, H. W.; Laajalehto, K. Surface States and Reactivity of Pyrite and Marcasite. App. Surf. Sci. 2001, 179, 222-229.

${ }^{20}$ Mattila, S.; Leiro, J. A.; Laajalehto, K. Surface XPS Core-Level Shifts of $\mathrm{FeS}_{2}$. Appl. Surf. Sci. 2003, 212-213, 97-100.

${ }^{21}$ Leiro, J. A.; Mattila, S.; Laajalehto, K. XPS Study of the Sulphur $2 \mathrm{p}$ Spectra of Pyrite. Surf. Sci. 2003, 547, 157-161.

${ }^{22}$ Kendelewicz, T.; Doyle, C. S.; Bostick B. C.; Brown, Jr., G. E. Initial Oxidation of Fractured Surfaces of $\mathrm{FeS}_{2}(100)$ by Molecular Oxygen, Water Vapor, and Air. Surf. Sci. 2004, 558, 80-88.

${ }^{23}$ Mattila, S.; Leiro, J. A.; Heinonen, M. XPS Study of the Oxidized Pyrite Surface. Surf. Sci. 2004, 566-568, 1097-1101.

${ }^{24}$ Ma, Y.; Lin, C. Microbial Oxidation of $\mathrm{Fe}^{2+}$ and Pyrite Exposed to Flux of Micromolar $\mathrm{H}_{2} \mathrm{O}_{2}$ in Acidic Media. Sci. Rep. 2013, 3, 1979.

25 Berlich, A. G.; Nesbitt, H. W.; Bancroft, G. M.; Szargan, R. Binding of Oxygen on Vacuum Fractured Pyrite Surfaces: Reactivity of Iron and Sulfur Surface Sites. Surf. Sci. 2013, 611, 60-66.

${ }^{26}$ Rimstidt, J. D.; Vaughan, D. J. Pyrite Oxidation: A State-of-the-Art Assessment of the Reaction Mechanism. Geochim. Cosmochim. Acta, 2003, 67, 873-880.

27 Rosso, K. M.; Vaughan, D. J. Sulfide Mineral Surfaces. Revs. Miner. Geochem. 2006, 61, 505-556.

${ }^{28}$ Rosso, K. M.; Vaughan, D. J. Reactivity of Sulfide Mineral Surfaces. Revs. Miner. Geochem. 2006, 61, 557-607.

29 Sit, H.-L. P.; Cohen, M. H.; Selloni, A. Interaction of Oxygen and Water with the (100) Surface of Pyrite: Mechanism of Sulfur Oxidation. J. Phys. Chem. Lett 2012, 3, 2409-2414.

30 Kresse, G.; Furthmüller, J. Efficiency of Ab-Initio Total Energy Calculations for Metals and Semiconductors Using a Plane-Wave Basis Set. Comput. Mat. Sci. 1996, 6, 15-50.; Kresse G.; Furthmüller, J. Efficient Iterative Schemes for Ab Initio TotalEnergy Calculations Using a Plane-Wave Basis Set. 
Phys. Rev. B 1996, 54, 11169-11186.

31 Perdew, J. P.; in Electronic Structure of Solids '91, edited by Ziesche, P.;Eschrig, H. Akademie Verlag: Berlin, 1991.; Perdew, J. P.; Wang, Y. Accurate and Simple Analytic Representation of the Electron-Gas Correlation Energy. Phys. Rev. B 1992, 45, 13244-13249.

32 Blöchl, P. E. Projector Augmented-Wave Method. Phys. Rev. B 1994, 50, 17953-17979.; Kresse, G.; Jouber, D. From Ultrasoft Pseudopotentials to the Projector Augmented-Wave Method. Phys. Rev. B 1999, 59, 1758-1775.

${ }^{33}$ Finklea, S. L.; Cathey, L.; Amma, E. L. Investigation of the Bonding Mechanism in Pyrite Using the Mössbauer Effect and X-Ray Crystallography. Acta Crystallogr., Sect. A 1976, 32, 529-537.

34 Stirling, A.; Bernasconi, M.; Parrinello, M. Defective Pyrite (100) Surface: An Ab Initio Study. Phys. Rev. B 2007, 75, 165406.

35 Makov, G.; Payne, M. C. Periodic Boundary Conditions in $\mathrm{Ab}$ Initio Calculations. Phys. Rev. B 1995, 51, 4014-4022.

${ }^{36}$ Hung, A.; Muscat, J.; Yarovsky, I.; Russo, S. P. Density-Functional Theory Studies of Pyrite $\mathrm{FeS}_{2}(100)$ and (110) Surfaces. Surf. Sci. 2002, 513, 511-524.

37 Mills, G.; Jónsson, H.; Schenter, G. K. Reversible Work Transition State Theory: Application to Dissociative Adsorption of Hydrogen. Surf. Sci. 1995, 324, 305-337.

38 Henkelman, G.; Jónsson, H. Improved Tangent Estimate in the Nudged Elastic Band Method for Finding Minimum Energy Paths and Saddle Points. J. Chem. Phys. 2000, 113, 9978-9985.

39 Bader, R. F. W. Atoms in Molecules: a Quantum Theory, Oxford University Press: New York 1990.

40 Tang, W.; Sanville, E.; Henkelman, G. A GridBased Bader Analysis Algorithm without Lattice Bias. J. Phys.: Condens. Matter. 2009, 21, 084204 .

41 The small difference between our results and those obtained by Selloni et al. ${ }^{29}$ can be explained by the different functionals (PW91 vs PBE), the different methodology (PAW vs ultrasoft pseudopotential schemes) and the different Brilluoin-zone samplings $(3 \times 3 \times 1$ Monkhorst-Pack k-point set vs $\Gamma$-point).

42 von Oertzen, G. U.; Skinner, W. M.; Nesbitt, H. W. $\mathrm{Ab}$ Initio and X-Ray Photoemission Spectroscopy Study of the Bulk and Surface Electronic Structure of Pyrite (100) with Implications for Reactivity. Phys. Rev. B 2005, 72, 235427.

${ }^{43}$ Herzberg, G. Spectra of Diatomic Molecules. In Molecular Spectra and Molecular Structure, 2nd Ed.; Krieger Publishing Co.: Malabar, FL, 1989; Vol. I.

${ }^{44}$ Nam, W. High-Valent Iron(IV)-Oxo Complexes of Heme and Non-Heme Ligands in Oxygenation Reactions. Acc. Chem. Res. 2007, 40, 522-531.

45 Sastri, C. V.; Park, M. J.; Ohta, T.; Jackson, T. A.;Stubna, A.; Seo, M. S.; Lee, J.; Kim, J.; Kitagawa, T.; Münck, E.; et al. Axial Ligand Substituted Nonheme $\mathrm{Fe}^{I V}=\mathrm{O}$ Complexes:? Observation of Near-UV LMCT Bands and $\mathrm{Fe}=\mathrm{O}$ Raman Vi- brations. J. Am. Chem. Soc., 2005, 127, $12494-$ 12495.; Pinakoulaki, E.; Daskalakis, V.; Ohta, T.; Richter, O.-M. H.; Budiman, K.; Kitagawa, T.; Ludwig B.; Varotsis, C. The Protein Effect in the Structure of Two Ferryl-Oxo Intermediates at the Same Oxidation Level in the Heme Copper Binuclear Center of Cytochrome c Oxidase. J. Biol. Chem., 2013, 288, 20261-20266.

46 Stirling, A.; Bernasconi, M.; Parrinello, M. Ab Initio Simulation of Water Interaction with the (100) Surface of Pyrite. J. Chem. Phys 2003, 118, 89178926.

47 Stirling, A.; Bernasconi, M.; Parrinello, M. Ab Initio Simulation of $\mathrm{H}_{2} \mathrm{~S}$ Adsorption on the (100) Surface of Pyrite. J. Chem. Phys 2003, 119, 49344939.

48 Nair, N. N.; Schreiner, E.; Marx, D. Glycine at the Pyrite-Water Interface: The Role of Surface Defects. J. Am. Chem. Soc. 2006, 128, 13815-13826.

49 Lengths of non-protonated ferryl-oxygen double bonds have been shown to be between 1.6-1.73 Å: Gumiero, A.; Metcalfe, C. L.; Pearson, A. R.; Raven, E. L.; Moody, P. C. E. Nature of the Ferryl Heme in Compounds I and II. J. Biol. Chem. 2011, 286, 1260-1268.

50 Nesbitt, H. W.; Bancroft, G. M. Pratt, A. R.; Scaini, M. J. Sulfur and Iron Surface States on Fractured Pyrite Surfaces. Am. Mineral. 1998, 83, 1067-1076.

${ }^{51}$ Becker, U.; Rosso, K. M.; Hochella Jr., M. F. The Proximity Effect on Semiconducting Mineral Surfaces: A New Aspect of Mineral Surface Reactivity and Surface Complexation Theory? Geochim. Cosmochim. Acta 2001, 65, 2641-2649.

52 Andersson, K.; Nyberg, M.; Ogasawara, H.; Nordlund, D.; Kendelewicz, T.; Doyle, C. S.; Brown Jr., G. E.; Pettersson, L. G. M.; Nilsson, A. Experimental and Theoretical Characterization of the Structure of Defects at the Pyrite $\mathrm{FeS}_{2}$ (100) Surface Phys. Rev. B 2004, 70, 195404.

53 Birkholtz, M.; Fiechter, S.; Hartmann, A.; Tributsch, H. Sulfur Deficiency in Iron Pyrite $\left(\mathrm{FeS}_{2-x}\right.$ and its Consequences for Band-structure Models. Phys. Rev. B 1991, 43, 11926-11936.

54 The dosage of $\mathrm{O}_{2}$ was estimated using the dimension of the vacuum region in the simulation box and the kinetic theory of gases. For a similar estimation see Ref. 5. 
For Table of Contents Only

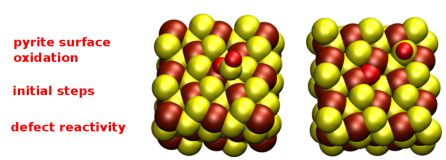

